\title{
Expulsion : genèse et pratique d'un contrôle en Allemagne (partie 1)
}

\section{Frank Paul Weber}

\section{OpenEdition \\ Journals}

\section{Édition électronique}

URL : http://journals.openedition.org/conflits/350

DOI : $10.4000 /$ conflits.350

ISSN : $1777-5345$

Éditeur :

CCLS - Centre d'études sur les conflits lilberté et sécurité, L'Harmattan

Édition imprimée

Date de publication : 15 octobre 1996

ISSN : 1157-996X

\section{Référence électronique}

Frank Paul Weber, «Expulsion : genèse et pratique d'un contrôle en Allemagne (partie 1) », Cultures \& Conflits [En ligne], 23 I automne 1996, mis en ligne le 15 mars 2006, consulté le 30 mars 2021. URL http://journals.openedition.org/conflits/350 ; DOI : https://doi.org/10.4000/conflits.350

Ce document a été généré automatiquement le 30 mars 2021

Creative Commons License 


\title{
Expulsion : genèse et pratique d'un contrôle en Allemagne (partie 1)
}

\author{
Frank Paul Weber
}

1 EXPULSION : GENESE ET PRATIQUE D'UN CONTRÔLE EN ALLEMAGNE Frank Paul WEBER $^{1}$

2 La pratique en Allemagne des expulsions d'étrangers se dérobe au regard de l'observateur qui n'y verrait qu'une des manifestations du pouvoir administratif de l'Etat moderne. Comment, en effet, analyser cette pratique qui implique, par exemple, des argumentaires opposés comme celui de la protection du marché national du travail et celui des opposants à une "Europe forteresse», une différenciation entre «nationaux» et "étrangers », un corpus de lois, d'ordonnances, des centres de rétention, un agencement particulier entre Etats fédérés et Etat fédéral, des acteurs aussi différents que l'administration d'un canton rural et des organisations internationales non-gouvernementales? Loin de n'être que le simple résultat des mécanismes d'une institution, cette pratique est le lieu de complexes relations de pouvoir mêlant des individus, des agences de contrôle, des diplomaties, des intérêts locaux et régionaux... Cette pratique apparaît comme un objet à la fois « résolument hétérogène, comportant des discours, des institutions, des aménagements architecturaux, des décisions réglementaires, des lois, des mesures administratives... ", dotée d'un lien entre ces éléments épars, formation enfin, qui, à un moment historique donné, a eu pour fonction majeure de répondre à une urgence. La pratique des expulsions d'étrangers en Allemagne a en effet toutes les caractéristiques de ce que Michel Foucault a nommé un dispositif ${ }^{2}$. Les conditions de la genèse de ce dispositif, son évolution sous divers régimes politiques sont les repères essentiels qu'il convient de détenir avant d'examiner quatre aspects actuels du dispositif: l'internement ou la rétention administrative, les droits de recours, son agencement fédéral et sa transnationalisation croissante.

GENESE DE L'EXPULSION

4 La ou les relations de pouvoir induites par la pratique de l'expulsion ont été largement déterminées par l'établissement progressif de différences juridiques. A la 
différenciation entre personnes "nocives, parasites » de la société et le reste de cette dernière, a succédé une autre différenciation: celle entre citoyens/nationaux et étrangers. Cette différenciation fut et est à la fois la condition et l'effet de la relation de pouvoir que représente l'expulsion ${ }^{3}$. L'expulsion concerne en effet jusqu'au XVIIIe siècle les vagabonds [Landstreicher], paresseux [arbeitsscheu], les mendiants ${ }^{4}$. Elle s'applique autant aux étrangers [Ausländer] qu'aux «indigènes " [Inländer]. Aux premières prises de position réprouvant l'expulsion des «nationaux" [Staatsangehörigen] apparues avec l'Aufklärung fit écho un ordre du ministère du roi de Prusse (4 janvier 1744) prévoyant le remplacement de l'expulsion de "nationaux » par leur réclusion en forteresse ou en pénitencier ${ }^{5}$. Puis tout au long de la première moitié du XIXe siècle vont se succéder mesures unilatérales de renonciation à l'expulsion de ses propres ressortissants (en Bavière en 1813), accords de réadmission réciproque de ses propres ressortissants expulsés par l'Etat contractant (décret du ministre de la police de Prusse du 10 oct. 1814, règlement repris par les gouvernements d'Hanovre, de Saxe-Weimar, de Braunschweig...). L'acceptation de ces deux règles (obligation de réadmission de ses ressortissants expulsés et renonciation à l'expulsion de ses ressortissants) va être parachevée par la signature de conventions multilatérales entre des Etats allemands. 32 Etats signent le traité de Gotha sur l'admission des expulsés (15 juillet 1851). Avec la création de la Confédération de l'Allemagne du Nord [Norddeutscher Bund] en juillet 1867 chaque Allemand est reconnu comme membre d'un Etat fédéré et bénéficie donc du droit de s'installer et de séjourner dans tout Etat fédéré en raison de l'« indigénat commun » propre à la Confédération (Article 3 de cette Constitution). Ainsi en évoluant vers des situations d'Etat de droit [Rechtsstaat], constitutionnels [Verfassungsstaat] les Etats allemands abandonnent des restrictions et contraintes pesant sur leurs ressortissants (les peines de réclusion ont pris, par exemple, le dessus sur les supplices, le bannissement) mais, parallèlement, par opposition ils élaborent peu à peu la figure de l'étranger, celui qui ne partage pas le même Heimatstaat. L'octroi de droits par l'Etat à ses "citoyens" entraîna parallèlement une juridicisation (Verrechtlichung), une codification de la différence entre ressortissants nationaux et étrangers. Ce passage du Polizeistaat au Rechtsstaat impliqua aussi que la situation des étrangers s'énonça dès lors avant tout en termes juridiques. Cette inclusion croissante des étrangers dans la sphère juridique ne marque ni une amélioration ni une détérioration a priori de leur statut: d'une part les étrangers étaient de plus en plus assujettis à un contrôle se perfectionnant, d'autre part la juridicisation ouvrait aussi des possibilités de recours face à des décisions administratives ${ }^{6}$. Du statut de quasi-égal en tant que sujet du même souverain, le vagabond étranger se voit rejeté non plus seulement en raison de sa situation sociale mais également parce qu'il n'est pas le "pauvre» du lieu ${ }^{7}$. La distinction fut ainsi nettement établie dans la législation sur les pauvres : en 1843 la loi prussienne sur la punition des vagabonds, des mendiants et des oisifs prévoyait qu'après avoir purgé leurs peines ces personnes devaient être, si elles étaient des «nationaux » [Inländer] placées dans un établissement de correction, s'il s'agissait d'étrangers [Ausländer] expulsées ${ }^{8}$. Avec le glissement de la responsabilité de l'aide aux pauvres des communes vers l'Etat, la question de l'appartenance [membership] à cet Etat se posa avec acuité. Cela fut aussi l'effet d'une différenciation croissante entre Etats. « Le terme et concept de Staatsangehörigkeit apparut pour la première fois dans des traités bilatéraux signés pour réguler et coordonner les pratiques d'expulsion" 9. La codification de la citoyenneté fut donc le produit d'une «nécessité » d'exclusion des migrants pauvres. 
Articulée au principe de résidence, cette appartenance citoyenne finit par tracer une ligne de partage, qui correspond aussi à celle du système étatique. Cette différenciation fut toutefois un long processus et ne supplanta pas immédiatement l'agencement antérieur, qui survécut sous la forme de nombreux statuts et normes. Ainsi si l'expulsion, le bannissement deviennent exclusivement applicables aux étrangers, des règlements prévoient encore ce type de sanction pour un « indigène ». Juridiquement la possibilité d'une expulsion entre Etats fédérés allemands demeurera jusqu'à l'ordonnance de 1934, le pouvoir national-socialiste la supprimant en même temps d'ailleurs que l'indépendance des Länder. La différenciation n'est cependant pas complète: l'expulsion est encore une sanction qui s'applique à des "citoyens". Bismarck conservera ce mode de sanction pour ses adversaires politiques : l'expulsion est une des sanctions prévues dans les lois d'exceptions visant les Jésuites (4 juillet 1872), les socialistes (21 octobre 1878), les catholiques (loi sur l'empêchement de l'exercice non-autorisé d'une charge d'Eglise, 4 mai 1874). La République de Weimar maintiendra aussi cette possibilité d'expulsion de ses propres citoyens dans la loi sur la protection de la République (21 juillet 1922), tout comme cela avait été le cas pour l'une des dernières lois d'urgence de l'Empire : la loi contre l'évasion fiscale (26 juillet 1918). Si la distinction entre les victimes, les « objets » de l'expulsion et les individus qui n'en couraient pas le risque ne correspondait pas encore "parfaitement" à la différenciation entre nationaux/citoyens et étrangers, cette différenciation inachevée n'était qu'un résidu de la coexistence de principes devant, à terme, s'exclure mutuellement. Deux principes de l'expulsion se faisaient, en effet, encore face: l'expulsion comme instrument pour se débarrasser d'éléments protestataires, "dangereux", asociaux et l'expulsion comme mesure ultime pour attester de la séparation entre les membres (et ceux dignes d'y adhérer, à terme, par naturalisation) d'une communauté et tous les autres qui n'en font pas ou ne méritent pas d'en faire partie. En Prusse le "passage de témoin » entre ces deux acceptions semble avoir été réalisé dans les années 1880. Au début de 1885, inquiété par la "polonisation» des confins orientaux de la Prusse, Bismarck décida d'expulser tous les "polonais-russes » (c'est-à-dire issus de la Pologne du Congrès placée sous contrôle russe) des quatre régions prussiennes frontalières de la Russie. Le décret signé par son Ministre de l'Intérieur ordonnait aux quatre Présidents de province de procéder à l'expulsion immédiate de tous les «transfuges" [Überläufer] russo-polonais séjournant sans autorisation dans leur district ${ }^{10}$. Quatre mois plus tard la mesure fut étendue aux Polonais originaires de Galicie, c'est-à-dire sujets de l'Empire austro-hongrois ${ }^{11} .27000$ des 39000 « transfuges » polonais dénombrés par les autorités prussiennes avaient déjà été expulsés à l'automne $1886^{12}$. Les Juifs représentaient un tiers des expulsés. Cela témoigne des objectifs multiples que le gouvernement prussien poursuivait avec ces expulsions. A la crainte de la possible renaissance d'un sentiment national polonais à l'intérieur même des frontières de l'Empire du fait du regroupement de Polonais issus de Galicie, de Russie et de Prusse, à la hantise d'une croissance des éléments catholiques s'ajoutait un antisémitisme se dissimulant sous des appellations sibyllines. Les Juifs d'Europe orientale avaient d'ailleurs été les premiers visés ${ }^{13}$ par des expulsions puisque dès 1881-1882 le gouvernement prussien avait entrepris de leur interdire l'accès au territoire prussien en leur refusant l'entrée ou en les expulsant. Les expulsions ne concernaient pas seulement les Juifs de Russie ou de Galicie séjournant dans les provinces frontalières mais aussi ceux de Berlin ${ }^{14}$. Ce rejet intervenait durant une période d'afflux en Prusse de Juifs de Russie où ils étaient victimes de pogroms 
déclenchés à la suite de l'assassinat d'Alexandre II (mars 1881). Bismarck avait cependant avec ces expulsions massives et une fermeture des frontières orientales sacrifié les intérêts économiques des provinces de l'Est pour sa politique de sécurité. Les propriétaires terriens de ces régions employaient en effet la main d'œuvre «bon marché » et docile des Polonais résidant dans la partie de la Pologne "associée » à l'Empire russe (Pologne du Congrès) ou dans celle octroyée à l'Autriche-Hongrie (la Galicie notamment) ${ }^{15}$. Ce flux migratoire transnational ayant été interrompu, ils ne pouvaient plus compenser la pénurie de main-d'œuvre provoquée par les migrations d'Allemands vers le « Nouveau Monde » ou à l'intérieur de l'Empire allemand vers les régions urbaines et industrielles de l'Ouest du pays. Aussi les associations de fermiers, les autorités régionales firent pression sur les autorités centrales de Berlin: «si l'agriculture doit continuer à exister, alors la seule issue est de ré-ouvrir les frontières closes et d'autoriser l'entrée aux travailleurs russes-polonais et galiciens, [qui peuvent résoudre la crise du marché du travail agricole] par leur zèle et leur simplicité ${ }^{16}$. Caprivi, le successeur de Bismarck, céda en novembre 1890 : la main d'œuvre venant de l'Est fut de nouveau officiellement autorisée dans l'agriculture et l'industrie ${ }^{17}$. Les pratiques développées lors de ces expulsions massives des années 1885-1890 indiquaient bien une limitation de l'application de l'expulsion aux non-ressortissants allemands. Les migrants qui n'avaient pas officiellement été naturalisés prussiens auparavant mais qui avaient servi dans l'armée prussienne ne furent pas expulsés, étant considérés comme Prussiens « de facto ». Par ailleurs, même s'ils faisaient l'objet d'une surveillance étroite de la police prussienne, les Polonais prussiens (notamment ceux travaillant depuis les années 1870 dans les mines de la Ruhr) ne furent pas non plus menacés par ces expulsions. Toutefois il s'agit plus d'une transformation de la notion d'expulsion que du remplacement des principes gouvernant celle-ci: dans l'image des expulsés des années 1880 se superposait en effet l'idée d'étranger et celle d'individus asociaux, vagabonds (cette dernière ayant été jusque-là l'idée-directrice des pratiques d'expulsion). En se «nationalisant » la notion d'expulsion a happé l'image de l'expulsé/l'expulsable, que les siècles antérieurs avaient façonnée. L'inscription «nationale» permettait aussi de procéder à l'expulsion de personnes visées pour d'autres raisons : ainsi les Juifs de Russie et d'Autriche-Hongrie réfugiés en Prusse étaient les victimes fréquentes de vagues d'expulsion ordonnées par les autorités prussiennes, qui les considéraient comme "subversifs" ${ }^{18}$. Au cours du XIXe siècle l'expulsion est une sanction qui se différencie de l'ensemble du corpus « répressif » de deux façons : d'une part elle est de plus en plus comprise par ceux qui l'ordonnent comme une mesure préventive, de « sécurité » que comme une peine. Il ne s'agit plus de « réparer, compenser un préjudice » mais d'empêcher de futurs dommages ${ }^{19}$. Cela se traduit d'ailleurs par deux types d'expulsion: celle effectuée à l'issue d'une condamnation pénale (sur la base du code pénal) et donc d'une décision de justice et celle purement administrative, ordonnée et réalisée par la police compétente (par exemple en Prusse la police locale, au Grand-Duché de Bade le Ministère de l'Intérieur) indépendamment de toute condamnation et en raison du danger que représentait l'étranger pour «la sécurité intérieure ou extérieure de l'Etat $"{ }^{20}$. D'autre part, elle ne concerne plus, au moins dans la pratique, que les étrangers et donc n'est plus une sanction qui s'applique à tous sans distinction.

5 Weimar Après la Première Guerre Mondiale, les pratiques d'expulsion sont étroitement liées aux premières mesures d'organisation du marché du travail. Les autorités de la République de Weimar utilisent tout un argumentaire économique pour justifier les 
expulsions auxquelles elles procèdent. Dans l'immédiat après-guerre, l'Office de démobilisation "organise » le transfert [Abtransport], c'est-à-dire l'expulsion, de travailleurs étrangers originaires d'Europe de l'Est au rythme de 30000 par mois ${ }^{21}$. L'idée centrale qui préside à ces expulsions est que ces travailleurs étrangers doivent céder leur emploi aux soldats allemands démobilisés revenant au pays ${ }^{22}$. Les dirigeants allemands soulignent cependant au même moment que l'agriculture ne saurait se passer des travailleurs étrangers et autorisent en novembre 1919 les propriétaires terriens à avoir recours à environ 50000 ouvriers agricoles polonais. Ce nouveau recrutement se déroule dans des conditions fort différentes de celles ayant court avant 1914 : l'entrée et l'emploi de la main-d'œuvre étrangère sont désormais clairement subordonnés à l'intérêt des travailleurs allemands ${ }^{23}$. Les fermiers devaient déposer une demande d'autorisation pour travailleurs agricoles étrangers. Une commission paritaire de représentants patronaux et syndicaux statuait ensuite sur les demandes. Les conventions collectives [Tarifverträge] devaient s'appliquer aux salariés allemands et étrangers. Cette priorité accordée aux salariés « nationaux » [Inländerprimat] est un des éléments de la régulation (c'est-à-dire bien souvent la protection) du marché du travail qui se met en place à l'époque ${ }^{24}$, consolidant ainsi l'Etat-providence allemand. Pour justifier les expulsions opérées durant la République de Weimar, les autorités indiquaient d'ailleurs, la plupart du temps, que l'étranger expulsé était indésirable, qu'il représentait un fardeau que l'Etat allemand ne pouvait supporter compte tenu de la crise du logement, du chômage et des difficultés alimentaires. Les étrangers susceptibles de faire appel à l'assistance aux chômeurs [Erwerbslosenfürsorge] ou à l'aide publique aux pauvres [öffentliche Armenpflege] étaient à expulser. Il en était de même pour ceux menaçant la sécurité et l'ordre publics, ou ayant fait l'objet d'une condamnation pénale. Ces motifs d'expulsion étaient, par exemple, énumérés dans les décrets d'expulsion édictés par la Prusse entre 1919 et 1923. Entre juin 1922 et septembre 193226000 personnes environ, dont au moins 3900 Juifs d'Europe de l'Est, furent expulsées de Prusse. Près des deux tiers des expulsés étaient des ressortissants polonais. C'est dans le contexte de l'occupation de la Ruhr et de l'hyperinflation que le nombre des expulsions atteignit son paroxysme (plus de 2900 personnes expulsées durant les six premiers mois de 1923). En Prusse, les procédures d'expulsion étaient du ressort de la police locale [Ortspolizei], après accord du gouverneur de province [Regierungspräsident]. Un délai oscillant, suivant les cas, entre trois jours et un mois ${ }^{25}$, était accordé afin de permettre un départ volontaire de l'expulsable. Ce délai ne suffisait généralement pas pour que la personne se munisse des documents nécessaires à son entrée dans un autre Etat. Des sursis supplémentaires pouvaient être octroyés suite à une plainte ou requête. La procédure pouvait ainsi s'étendre sur deux ou trois $a_{n}{ }^{26}$. Bien que les décrets prévoyaient les cas où des étrangers ne pouvaient faire l'objet d'un arrêté d'expulsion (comme, par exemple, les étrangers entrés illégalement en Prusse mais y demeurant depuis au moins quatre ans), la police ignorait souvent ces limitations et engageait une procédure d'expulsion. Les expulsions prononcées ne pouvaient pas toujours être exécutées. En Rhénanie occupée par les Alliés, les expulsions d'étrangers nécessitaient l'aval de la Haute Commission Interalliée des Territoires Rhénans. Les principaux obstacles à l'exécution des arrêtés d'expulsion résidaient toutefois dans les limites fixées par le droit international public (notamment avec l'interdiction d'expulser déserteurs, réfractaires et apatrides) et surtout dans la réticence, voire le refus, des Etats d'origine d'accueillir leurs propres ressortissants sur leur territoire ${ }^{27}$. De plus, il faut garder à l'esprit que dans les années qui suivirent la fin 
de la Première Guerre Mondiale, la plupart des frontières des pays concernés n'étaient pas encore établies. La formation de nouveaux Etats en Europe centrale et orientale suscitait par ailleurs une nouvelle immigration vers l'Allemagne ${ }^{28}$. Aussi pour les étrangers originaires de Galicie, d'Ukraine, de Lituanie et de Pologne ${ }^{29}$, la nationalité "d'après-guerre " n'était pas toujours clairement avérée. Si, sous Weimar, la plupart des Länder allemands (la Prusse mais aussi la Saxe, le Mecklembourg-Schwerin, la Bavière, Brême...) procédaient à des expulsions d'étrangers, les pratiques étaient différentes d'un Etat fédéré à l'autre. Les majorités politiques prévalant dans chaque Land expliquent en partie cette hétérogénéité ${ }^{30}$. Partout des prétextes " économiques » étaient mis en avant pour justifier les expulsions. Bien souvent, à mots couverts, les Juifs d'Europe de l'Est étaient les principaux visés. La Bavière fut l'Etat qui alla le plus loin en ce sens : de 1919 à 1921 les dirigeants bavarois décrétèrent plusieurs expulsions d'étrangers qui touchèrent plusieurs centaines de Juifs. En octobre 1923, von Kahr, investi des pleins pouvoirs par le gouvernement de Bavière, engagea la vague d'expulsion la plus spectaculaire de la période Weimar. Une centaine de Juifs, étrangers mais aussi originaires du pays de Bade et de Prusse, furent expulsés. Simultanément avec la notification d'expulsion était ordonnée la mise sous séquestre du logement des expulsés, voire le cas échéant de leur entreprise. Les instructions données par Kahr au Ministère de l'Intérieur confirment, s'il en était besoin, que ces mesures furent draconiennes : «Un comportement dommageable à la vie économique est une raison suffisante pour procéder à l'expulsion d'étrangers. Si une expulsion est prononcée à l'encontre du chef de famille, alors cette mesure doit être étendue aux membres de sa famille vivant dans son ménage... Les appartements et logements des étrangers expulsés sont considérés comme saisis ${ }^{31}$. Cette brutalité était loin d'être l'exception dans une Bavière secouée par les mouvements d'extrême-droite se livrant à une surenchère d'antisémitisme. Le NSDAP naissant en avait fait l'essentiel de son programme et c'est durant ce même automne 1923 que Hitler tenta un putsch. Les rapports entre les pouvoirs et les étrangers furent l'objet d'une juridicisation croissante sous la République de Weimar. Pour une part, cette soumission croissante du séjour des étrangers à des principes juridiques [Verrechtlichung] coïncida avec la juridicisation de la sphère du travail. En Prusse, cette inscription des étrangers dans le droit (qui s'ajoutait à leur définition antérieure également par le droit: celui de la nationalité) aboutit, en avril 1932, à l'adoption d'un règlement de police portant sur le traitement des étrangers [Polizeiverordnung über die Behandlung der Ausländer] ${ }^{32}$. Ce décret remplaçait une législation dispersée (près de deux cents décrets furent « unifiés»), ce qui semblait procéder à une simplification administrative. De plus, si la " police des étrangers » [Fremdenpolizei] était - comme déjà sous l'Empire wilhelmien de la compétence de l'Empire [Reich], aucune loi fédérale réglementant le séjour des étrangers dans l'Empire n'avait jamais été adoptée. La législation des différents Länder était donc déterminante, celle de la Prusse plus particulièrement faisant souvent office de modèle pour les autres Länder et en raison, notamment, de sa dimension démesurée par rapport aux autres Etats fédérés. Ce règlement de police ne correspondait pourtant pas à l'octroi de droits objectifs aux étrangers. Aussi la comparaison avec l'évolution de la législation envers les étrangers dans les pays occidentaux d'après 1945 n'est pas ici pertinente. Cette unification des règles appliquées aux étrangers en Prusse s'adressait exclusivement à l'administration, à qui elle devait fournir des critères précis pour autoriser ou refuser un séjour en Prusse, pour décider d'une reconduite à la frontière, d'une expulsion... C'est ce règlement qui introduit le permis de séjour 
[Aufenthaltserlaubnis](§ 3), nécessaire à tout étranger demeurant plus de six mois en Prusse ou voulant y exercer un métier. Il était émis par la police du canton [Kreispolizeibehörde]. Son pendant répressif était également exposé dans ce règlement de 1932. Les différents motifs de l'expulsion, qui était de la compétence de la police du Land, y étaient dénombrés: la condamnation pour crime ou délit, la détention ordonnée à la suite d'un jugement, l'activité anti-étatique [staatsfeindlich] contre l'Empire ou un Land, la menace aux relations extérieures de l'Empire, la menace à la santé publique et aux mœurs, le fait de percevoir une assistance publique [öffentlicher Fürsorge] en refusant de quitter volontairement le territoire, la mendicité, le vagabondage, le séjour illégal sur le territoire prussien ainsi que les motifs prévus pour une expulsion de l'Empire [Reichsverweisung]. Pour la première fois apparaissait, de façon non équivoque, les cas où l'expulsion ne pouvait être ordonnée: lorsque l'étranger résidait depuis plus de dix ans dans l'Empire ou lorsqu'il avait moins de quinze ans. C'est cette catégorie juridique qui a été, après 1945, considérablement élargie dans la législation (ouest-) allemande sur les étrangers et qui, par conséquent, est un des éléments distinctifs entre les normes juridiques antérieures et celles postérieures à la Seconde Guerre Mondiale ${ }^{33}$. Par ailleurs l'expulsion des résidents étrangers depuis plus de cinq ans était rendue plus difficile. Il n'en reste pas moins que si ce décret d'avril 1932 représentait une clarification du droit des étrangers en Prusse, il ne constituait en aucune manière un droit [Rechtsanspruch] que les étrangers auraient pu revendiquer. Aucun recours devant un tribunal indépendant (i.e. un tribunal administratif) n'était possible : il ne pouvait être fait appel de la décision - et notamment d'un arrêté d'expulsion - que par réclamation [Beschwerde] auprès des autorités administratives. Le Ministre de l'Intérieur, i.e. celui de la police, du Land statuait en dernier ressort sur ces réclamations. Cet aspect illustre bien le souci des rédacteurs de ce règlement de ne limiter en aucune manière les compétences de l'Etat basées sur sa souveraineté territoriale au profit des étrangers résidant en Prusse ${ }^{34}$. Ce règlement de police avait ainsi l'avantage pour l'administration prussienne de maintenir son pouvoir discrétionnaire en matière de séjour, d'expulsion des étrangers : aux dires d'un conseiller du Ministère de l'Intérieur prussien de l'époque ce passage d'une directive administrative à une norme juridique permettait de disposer d'une "forme élastique» permettant de pallier, dans la pratique, aux insuffisances sans devoir toutefois avoir recours à un lourd appareil de lois. Il était possible, dès lors, d'adapter les pratiques d'expulsion aux «conditions politiques et économiques changeantes du moment ». Ce fut, par exemple, le cas avec les fluctuations du marché du travail ${ }^{35}$. Le Ministre de l'Intérieur pouvait en effet modifier tout ou partie du règlement en vigueur par simple décret.

6 La période nazie Après l'arrivée au pouvoir de Hitler les expulsions devinrent un des instruments les plus familiers de la machine de mort nationale-socialiste. L'expulsion de Juifs représenta une première étape (provisoire) de la politique d'extermination de la dictature nazie. Si, d'une certaine façon, le principe - développé au XIXe siècle - de non-expulsion de ses propres ressortissants fut maintenu par le régime nazi, les pratiques d'expulsion, entre autres, indiquaient une aggravation et une systématisation des anciens principes du Polizeistaat. L'expulsion et, plus tard, l'exécution des individus soi-disants "asociaux» en était l'épicentre. Parallèlement les Nazis procédaient à la privation croissante des droits des Juifs ${ }^{36}$. N'étant plus considérés comme des citoyens égaux en droit (et donc non-expulsables), toute protection juridique leur fut ainsi retirée, notamment en cas d'expulsion. Décisives furent, à cet 
égard, les "lois de Nuremberg ", et en particulier la «loi de citoyenneté du Reich » [Reichsbürgergesetz] qui divisait les citoyens allemands en "ressortissants" [Staatsangehörige] et «citoyens du Reich» [Reichsbürger] : «Seuls les «Citoyens du Reich " [Reichsbürger] devaient être citoyens [Staatsbürger] au plein sens du terme avec tous les droits politiques, étant "ressortissants [Staatsangehörige] de sang allemand ou apparenté ». Les Juifs furent ainsi dégradés de nationaux en citoyens de seconde classe, c'est-à-dire en définitive en sujets [Untertanen] " ${ }^{37}$. L'objectif de centralisation, d'unité étatique suivi par les nazis abolit en 1934, à la suite de la suppression des Länder, la distinction entre expulsion hors d'un Land [Landesverweisung] et celle en dehors du Reich. L'expulsion devint l'apanage du pouvoir central. La loi sur les expulsions adoptée (Reichsverweisungsgesetz de mars 1934, complétée par un décret d'application en mai) reprit l'essentiel des dispositions du règlement de police sur les étrangers pris par la Prusse en 1932. L'expulsion pour motifs politiques était étendue $(\$ 2,3){ }^{38}$. Le décret de "police des étrangers " [Ausländerpolizeiverordnung] d'août 1938 étendit de la même façon les stipulations du règlement prussien de $1932^{39}$ à l'ensemble du territoire du Troisième Reich, constituant ainsi la première législation fédérale allemande en matière d'entrée et de séjour des étrangers. Avant que l'expulsion devienne, en 1938, un programme systématique ${ }^{40}$, les pratiques des organes nazis et en particulier de la Gestapo avaient depuis 1934 aboli les différences entre exil volontaire, exil forcé, fuite et expulsion. A partir de 1938 la dictature nazie eut recours à une sorte de dialectique de terreur afin de réaliser ses objectifs de persécution envers les Juifs : la mise en détention croissante en camp de concentration, notamment à Dachau, était utilisée aussi comme moyen de pression pour accélérer et contraindre à l'exil ${ }^{41}$. Fin octobre 1938 plus de 17000 Juifs de nationalité polonaise vivant en Allemagne furent l'objet d'un arrêté d'expulsion et donc conduits par la police allemande en camion et en train à la frontière germanopolonaise. Cette politique "combinée » d'expulsions, de détentions et d'émigration forcée fut continuée jusque vers $1941^{42}$, lorsque le régime nazi, avec les déportations massives vers l'Est et l'extermination des Juifs d'Europe, atteint la dernière étape de son programme.

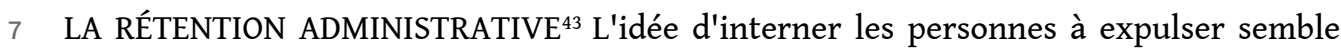
avoir fait lentement son chemin dans l'esprit des dirigeants prussiens et allemands. Les premiers cas d'internement d'individus en voie d'expulsion étaient des arrangements "de facto ", les expulsés étant rassemblés dans des lieux précaires car leur expulsion, en définitive leur réadmission dans leur pays d'origine, était bloquée. Lors des expulsions massives des années 1885-1890 ce fut ainsi le cas lorsque les autorités russes refusèrent d'admettre sur leur territoire des Polonais et des Juifs, sujets russes. Ils étaient rassemblés aux postes frontières dans des granges, des baraquements ${ }^{44}$. $C^{\prime}$ est envers les Juifs d'Europe de l'Est [Ostjuden] que plusieurs personnalités politiques de la République de Weimar imaginèrent et réalisèrent les premiers internements: le 16 décembre 1919, le Ministre de l'Intérieur de Prusse, Wolfgang Heine, envisagea devant le Parlement prussien l'expulsion hors des villes de tous les Juifs de l'Est "indésirables» et leur internement dans des "camps spéciaux de concentration ». Quelques mois plus tard le Général von Seeckt émit le même souhait et 250 Ostjuden furent placés dans un camp militaire à Wunsdorf près de Zossen. L'internement des Juifs d'Europe de l'Est était de plus en plus proposé comme une possible alternative à leur expulsion, l'expulsion étant «moralement injustifiable» et de toute façon éventuellement impossible à exécuter en raison du refus de réadmission de la Pologne. 
Dans une note du Ministère des Affaires étrangères [Auswärtiges Amt], il était proposé de " placer les éléments en camp d'internement, car ainsi il est possible d'endiguer la crise du logement et de décourager toute nouvelle immigration non autorisée. Il serait possible de convaincre à l'émigration [Auswanderung] les internés et un renvoi massif serait facilité. » ${ }^{45}$. En janvier 1921, le Ministre prussien de l'Intérieur, Carl Severing, engagea une politique officielle d'internement des Ostjuden qui ne fut levée qu'en décembre $1923^{46}$. Des camps à Stagard et Cottbus furent utilisés ${ }^{47}$. Avec l'arrivée au pouvoir d'Hitler et l'établissement de la dictature nazie, ces idées d'internement changèrent d'échelle : l'internement devint une des pièces maîtresses pour écarter et éliminer ceux que la "Weltanschauung" nazie qualifiait d'asociaux, d'ennemis politiques. Alors que les tentatives d'internement des premières années de la République de Weimar apparurent plutôt isolément, non-coordonnées, la détention de l'«étranger» construit par l'idéologie nationale-socialiste était résolument méthodique. Le pouvoir nazi systématisa la détention arbitraire et chercha, en partie, à lui octroyer un semblant de légalité. L'un des instruments de terreur utilisés par la Gestapo fut la "Schutzhaft ", " détention par mesure de sécurité ", dans un camp de concentration. Tous les adversaires du régime national-socialiste en furent victimes, dès 1933 avec les internements massifs des opposants politiques. Un décret du Président du Reich (28.2.1933) portant sur l'incendie du Reichstag fournit un semblant de fondement juridique à ces internements ${ }^{48}$. Himmler introduisit un autre instrument répressif avec la détention "préventive » [Vorbeugungshaft] . Un décret du Ministre de l'Intérieur du Reich [Runderlaß du 14.12.1937] « légalisa » a posteriori l'envoi en camp de concentration des personnes au comportement soi-disant « asocial » : c'est-à-dire, d'après les dirigeants nazis, les «mendiants, les vagabonds, les prostituées, les ivrognes, les oisifs, les tziganes " ${ }^{49}$. La première clause précise portant sur les centres de rétention [Abschiebungshaft] apparut dans le décret sur la police des étrangers [Ausländerpolizeiverordnung d'août 1938], dont l'article 7 stipulait: "en vue de garantir l'expulsion, un étranger peut être placé en centre de rétention» (§ 7, Abs.5, p. 2).

Lire la suite

\section{NOTES}

1. Allocataire de recherche (Centre d'Etudes et de Recherches Internationales), IEP Paris, Doctorant associé au Centre Marc Bloch, Berlin.

2. Michel Foucault, Dits et Ecrits, Paris, Gallimard, 1994, Tome III, p.299.

3. sur le système de différenciations, Cf. Michel Foucault, "Le pouvoir, comment s'exerce-t-il ?", in Hubert Dreyfus/Paul Rabinow, Michel Foucault, un parcours philosophique, Paris, Gallimard, 1984, [Folio-Essais, n²04] pp.316-317.

4. Cf. article "Abschiebung", Der Große Brockhaus, Leipzig, F.U. Brockhaus, Band 1, 1928, pp. 48-49.

5. Cf. Werner Kobarg, Ausweisung und Abweisung von Ausländern, Berlin, Dr. Walther Rothschild, 1930, p. 15 ; Helmut Erlanger, Interterritoriale Ausweisung und Weimarer 
Reichsverfassung, Stuttgart, Ferdinand Enke Verlag, 1932, p. 10 ; Heinz Nestmann, Die Reichsverweisung in Geschichte und Gegenwart, Dresde, Verlag M. Dittert \& Co., 1936, p. 20.

6. Cf. Nestmann, op. cit., p. 25.

7. Cf. le lien indissoluble de l'individu à un "Heimatsort" (lieu d'origine) stipulé par la loi prussienne sur les pauvres de 1842. Ce lien permettait de bénéficier de l'aide aux pauvres (les communes, pour des raisons financières, ne voulaient s'obliger qu'au seul soutien de "leurs" pauvres). Antje Kraus met en relief la conjonction du droit de la patrie [Heimatrecht] et celui de soutien aux pauvres, posant en partie ce dernier comme la conséquence du premier : "Die rechtliche Lage der Unterschicht im Übergang von der Agrar- zur Industriegesellschaft, in Hans Mommsen/Winfried Schulze (Hrsg.), Vom Elend der Handarbeit, Probleme historischer Unterschichtenforschung, KlettCotta, 1981, p.247.

8. Cette loi du 6 janvier 1843 reprenait, en les précisant, les attendus de l'art. 4 de l'Allgemeines Landrecht für die preußischen Staaten, du 1er juin 1794, qui stipulait l'expulsion des mendiants étrangers.

9. Rogers Brubaker, Citizenship and Nationhood in France and Germany, Cambridge/ London, Harvard University Press, 1992, p.68

10. Décret du 26 mars 1885 ; Cf. Neubach, op. cit., p.33.

11. Neubach, op. cit., p.60.

12. Neubach, op. cit., p.124

13. Neubach, op. cit., p.22.

14. En 1884 le nombre des Juifs expulsés de Berlin (662) dépassait celui des Juifs expulsés des quatre provinces de l'Est prussien (677). Cf. Neubach, op. cit., p.21.

15. Cf. Klaus Bade, "Transnationale Migration und Arbeitsmarkt im Kaiserreich : Vom Agrarstaat mit starker Industrie zum Industriestaat mit starker agrarischer Basis" in T. Pierenkemper/ R. Tilly (Hg.),Historische Arbeitsmarktforschung. Entstehung, Entwicklung und Probleme der Vermarktung von Arbeitskraft., Göttingen, Vandenhoeck \& Ruprecht [Kritische Studien zur Geschichtswissenschaft, Band 49], pp. 182-211.

16. Rapport du Regierungspräsident d'Oppeln [Haute-Silésie] à l'Oberpräsident de Breslau. 22 octobre 1890. K. Bade, 1982, op. cit., p.140.

17. Cf. Neubach, op. cit., p.135

18. Les Juifs réfugiés à Berlin étaient particulièrement visés, quelquesoit leur statut ou leurs activités (étudiants, ouvriers, petits marchands...) : Cf. Jack WERTHEIMER, Unwelcome Strangers, East European Jews in Imperial Germany, New York/Oxford, Oxford University Press, 1987, pp.60-63.

19. Cf. Nestmann, op. cit., p.11.

20. Voir, par exemple, le code pénal prussien du 14 avril 1851 (basé sur le code pénal français du 22 février 1810), § 120 Abs 2 et le code pénal du Grand-Duché de Bade de 1845 (art.18).

21. Rapport du député Schiele à propos de la discussion sur les travailleurs agricoles (Commission "économie" de l'Assemblée nationale), mars 1919, cité in Knut Dohse, Ausländische Arbeiter und bürgerlicher Staat, Genese und Funktion von staatlicher Ausländerpolitik und Ausländerrecht, Vom Kaiserreich bis zur Bundesrepublik Deutschland, Berlin, EXpress Edition, 1985, p.90.

22. Cf. Dohse, op. cit., pp.88-89. 
23. Cette évolution est commune, à l'époque, à d'autres pays européens, notamment la France : Cf. Gérard Noiriel, La tyrannie du national. Le droit d'asile en Europe 1793-1993, Paris, Calmann-Lévy, 1991, p.90.

24. Cf. représentation paritaire, intervention de l'Etat dans la régulation du marché du travail, introduction de l'assurance-chômage...

25. Cf. Trude Maurer, Ostjuden in Deutschland, 1918-1933, Hamburg, Hans Christians Verlag, 1986, p.386.

26. Cf. le cas Luszczanowski rapporté par Trude Maurer, op. cit., pp.380-381.

27. Il est notamment question ici d'accords de réadmission. Voir ci-dessous pour la présentation de ces accords.

28. Cf. Reiner Pommerin, "Die Ausweisungen von "Ostjuden" aus Bayern 1923", Vierteljahrshefte für Zeitgeschichte, Heft 3, 1986, p.317.

29. Cf. les conflits territoriaux à propos de Vilnius, de la Haute Silésie, des frontières orientales de la Pologne...

30. Cf. par exemple la corrélation entre les changements de majorité en Bavière en 1920, en Mecklembourg-Schwerin en 1924 et des opérations massives d'expulsion au même moment dans ces régions.

31. Reiner Pommerin, op. cit., p.315.

32. Cf. Nestmann, op.cit., pp.54-58 ; Volkmar Götz, "Polizei und Polizeirecht", in Kurt G.A. Jeserich, Hans Pohl, Georg-Christoph von Unruh (Hrsg.), Deutsche Verwaltungsgeschichte, Band 4 : Das Reich als Republik und in der Zeit des

Nationalsozialismus, Stuttgart, Deutsche Verlags-Anstalt, 1985, p.412 ; Dohse, op. cit., pp.107-112.

33. Voir ci-dessous notre présentation des droits de recours.

34. Cf. Dohse, op. cit., p.110.

35. Cf. Ulrich Herbert, Geschichte der Ausländerbeschäftigung in Deutschland 1880 bis 1980, Saisonarbeiter, Zwangsarbeiter, Gastarbeiter, Berlin/Bonn, Dietz, 1986, p.118. 36. Cela pouvait également consister à déchoir un citoyen allemand de sa nationalité [Ausbürgerung], pratique qui survécut au nazisme, notamment en RDA, lorsque les dirigeants est-allemands (suivant aussi une pratique soviétique) privèrent, en 1976, le dissident Wolf Biermann de sa nationalité.

37. Walther Hofer, "Stufen der Judenverfolgung im Dritten Reich 1933-1939", in Herbert A. Strauss/Norbert Kampe (Hrsg.), Antisemitismus, Von der Judenfeindschaft zum Holocaust, Frankfurt am Main/New York, Campus Verlag, 1985, p.179.

38. Cf. Nestmann, op.cit., p.71.

39. Cf. Götz, op. cit., p.1028.

40. Cf. Jacob Toury, "Ein Auftakt zur »Endlösung« : Judenaustreibungen über nichtslawische Reichsgrenzen, 1933-1939.", in Ursula Böttner/Werner Johe/Angelika Voß (Hrsg.), Das Unrechtsregime. Internationale Forschung über den Nationalsozialismus. Band 2 : Verfolgung - Exil - Belasteter Neubeginn, Hamburg, Hans Christians Verlag, 1986 [Festschrift für Werner Jochmann ; Hamburger Beiträge zur Sozial- und Zeitgeschichte ; Bd. 22] pp.168-169.

41. « Le placement en camp de concentration devint en novembre 1938, avec une soudaineté brutale, le moyen le plus important pour contraindre à l'émigration. (...) Fin 1938, il était nettement indiqué dans le rapport de l'Office principal du SD [SDHauptamt] : « Afin de renforcer la contrainte d'émigration, environ 25000 hommes juifs seront au même moment, en partie provisoirement, transférés en camp de concentration. ", Jacob Toury, op. cit., p.178. 
42. Cf. Juliane Wetzel, "Auswanderung aus Deutschland", in Wolfgang Benz (Hrsg.), Die Juden in Deutschland, 1933-1945. Leben unter nationalsozialistischer Herrschaft, Munich, Verlag C.H. Beck, 1988, pp.430-431.

43. "Abschiebegewahrsam".

44. Cf. Neubach, p.80.

45. Aufz. Ostjuden, Pol IV, 2.5.1920, Politisches Archiv des Auswärtigen Amts, Bonn, cité in Pommerin, op.cit., p.320.

46. D'après Pommerin, les mesures d'internement furent, dès novembre 1921, de nouveau limitées aux repris de justice et aux "éléments anti-étatiques", Pommerin, op.cit., p.321.

47. «The pressures from German public opinion even led Minister Heine to declare in the Prussian Landtag on 16 December 1919 that he was considering expelling all "undesirable" Ostjuden from the cities and interning them in special concentration camps. ", John P. Fox, "Weimar Germany and the Ostjuden, 1918-1923 : acceptance or expulsion ?" in Anna Bramwell, Refugees in the Age of Total War, London, Unwin Hyman, 1988, pp.60-61 et note 54. Cf. Pommerin, op.cit., p.319-321 : il indique, pour sa part, l'opposition d'Heine à l'antisémitisme et à l'expulsion des Juifs d'Europe de l'Est : «malgré le renforcement de l'antisémitisme et la situation difficile persistante sur le marché du travail, le Ministre prussien de l'Intérieur, Wolfgang Heine, se vit obligé en novembre 1919 de veiller à ce que les Juifs d'Europe de l'Est ne soient pas expulsés de Prusse. »

48. L'appareil d'extermination national-socialiste ne pouvant, ni ne devant être présenté succinctement, on se reportera sur ces questions à ces ouvrages pour une première approche : Raul Hilberg, La destruction des Juifs d'Europe, Paris, Fayard/ Gallimard, 1988 (notamment Tome I, p.180) ; Martin Broszat, L'Etat hitlérien, l'origine et l'évolution des structures du Troisième Reich, Paris, Fayard, 1985 [trad. fr. de Der Staat Hitlers, München, 1970], pp.127-129 ; Wolfgang Sofsky, Die Ordnung des Terrors : Die Konzentrationslager, Frankfurt/Main, Fischer Verlag, 1993 [trad ; fr. :

L'organisation de la terreur, Paris, Calmann-Lévy, 1995] ; Reinhard Rürup, Topographie des Terrors, Berlin, Topographie des Terrors Stiftung, 1989, pp.99-106.

49. Cf. Martin Broszat, Der Staat Hitlers, op.cit., pp.416-417 et Götz, op.cit., p.1021.

INDEX

Mots-clés : étrangers, expulsions, contrôle Index géographique : Allemagne 